\title{
A combined method for the treatment of grade IV internal haemorrhoidal disease
}

\author{
Georgios-loannis Verras ${ }^{1}$, Francesk Mulita ${ }^{1}$, George Theofanis ${ }^{1}$, Levan Tchabashvili ${ }^{1}$, loannis Perdikaris ${ }^{1}$, \\ Theano Perri ${ }^{1}$, Charalampos Kaplanis ${ }^{1}$, Dimitrios Filis ${ }^{2}$ \\ ${ }^{1}$ Department of Surgery, General University Hospital of Patras, Patras, Greece \\ 2Department of Surgery, "St. Andrew" General Hospital, Patras, Greece
}

Gastroenterology Rev 2023; 18 (1): 123-124

DOI: https://doi.org/10.5114/pg.2022.112776

\begin{abstract}
Address for correspondence: Dr. Francesk Mulita, Department of Surgery, General University Hospital of Patras, Patras, Greece, e-mail: oknarfmulita@hotmail.com
\end{abstract}

A 48-year-old patient presented acutely in our emergency department, reporting a fainting episode that took place several minutes prior, as well as continuous rectal blood loss. The patient's prior medical history included known grade IV haemorrhoidal disease for the past 33 years, as well as one more instance of hospitalization for haemorrhoid-associated blood loss. Examination of the patient's vital signs revealed a blood pressure of $98 / 67 \mathrm{~mm} \mathrm{Hg}$ and a pulse rate of $68 \mathrm{bpm}$, while the respiratory rate and temperature were within the normal limits. Anorectal inspection revealed massive, grade IV internal haemorrhoidal disease, with numerous prolapsed and ulcerated haemorrhoids as well as active bleeding sites. Digital palpation of the rectum returned stool of normal consistency, as well as ample fresh blood. Complete blood count report returned hemoglobin $(\mathrm{Hb})$ values of $7.0 \mathrm{~g} / \mathrm{dl}$ and hematocrit $(\mathrm{Hct})$ of $24.3 \%$ in addition to marked neutrophilia (73.9\%), indicating severe acute blood loss.

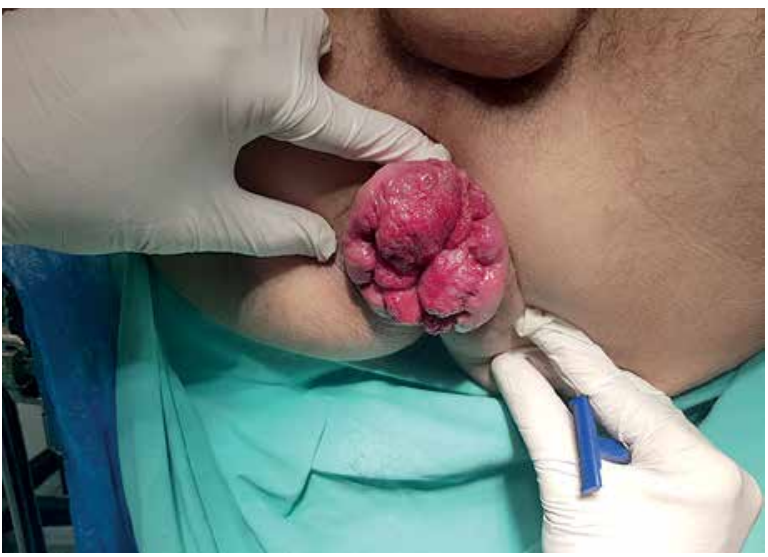

Figure 1. Preoperative view of the patient's haemorrhoidal disease
The patient was immediately administered 1 I of rapid infusion of crystalloid solution and received transfusion of 1 unit of crossmatched packed red blood cells ( $p R B C$ ), in order to achieve haemodynamic stability. An emergency recto-sigmoidoscopy was performed in order to establish the absence of other causes of rectal bleeding, which confirmed the presence of massive internal haemorrhoids on the 5, 7, and 11 o'clock positions, with active bleeding. The patient was immediately admitted to the surgical ward and underwent emergency surgical treatment for internal haemorrhoidal disease (Figure 1). The patient was placed at the lithotomy position under general anaesthesia, and a large-diameter Fansler anoscope was used. The technique employed here was a combination of Milligan-Morgan haemorrhoidectomy with additional haemorrhoidal suture ligation. Postoperatively, the patient received an additional 2 units of pRBC, remained stable, and was discharged after 2 days. The patient was in a good condition in his follow-up (Figure 2).

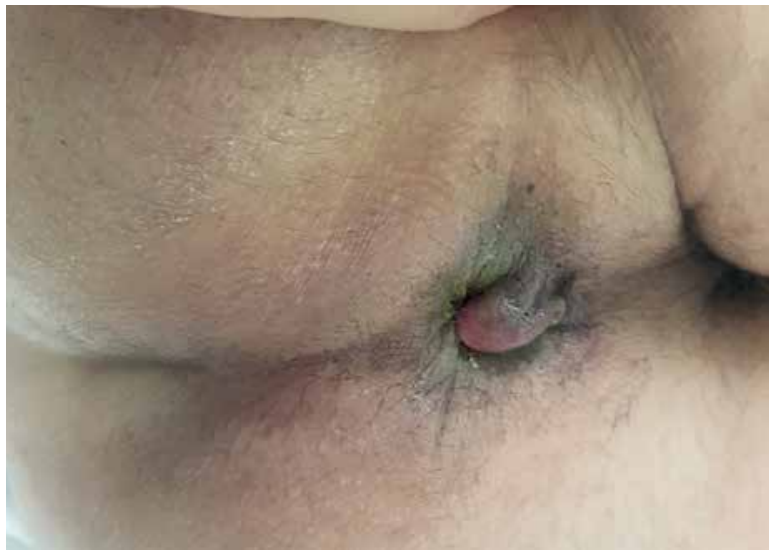

Figure 2. Postoperative image of the patient 20 days after the procedure 
Haemorrhoidal disease affects about $5 \%$ of the general population. Approximately $60 \%$ of the aforementioned people experience haemorrhoid-related symptoms, and from those only a small minority will experience symptoms of haemorrhagic shock, attributed to haemorrhoidal bleeding [1, 2]. After initial assessment and haemodynamic resuscitation, emergency surgical treatment is the preferred option for massive haemorrhoidal bleeding [1, 3, 4]. Complex cases such as ours often require a combination of available techniques in order to achieve bleeding control. Haemorrhoidectomy using the Milligan-Morgan approach is useful, not only allowing for definitive management of patients with chronic grade IV haemorrhoidal disease, but also with lower recurrence rates $[5,6]$.

The use of LigaSure ${ }^{\mathrm{TM}}$ devices is also related to lower postoperative complication rates and less pain [6]. Unfortunately, despite being an optimal solution for the treatment of complex haemorrhoidal disease, our institution could not provide access to LigaSure ligation devices, and simple sutured ligation was employed instead. In fact, despite the complexity of our case and the lack of optimal instrumentation, the utilization of simple ligation and haemorrhoidectomy allowed for an optimal result for our patient, proving that the proper technique is the primary contributor to good outcomes, even in the most challenging cases.

\section{Acknowledgments}

Georgios-loannis Verras and Francesk Mulita - equal contribution.

\section{Conflict of interest}

The authors declare no conflict of interest.

\section{References}

1. Sneider EB, Maykel JA. Diagnosis and Management of symptomatic hemorrhoids. Surg Clin North Am 2010; 90: 17-32.

2. Riss S, Weiser FA, Schwameis K, et al. The prevalence of hemorrhoids in adults. Int J Colorectal Dis 2012; 27: 215-20.

3. Sun Z, Migaly J. Review of Hemorrhoid disease: presentation and management. Clin Colon Rectal Surg 2016; 29: 22-9.

4. Lohsiriwat V. Anorectal emergencies. World J Gastroenterol 2016; 22: 5867.

5. Arbman G, Krook H, Haapaniemi S. Closed vs. open hemorrhoidectomy: is there any difference? Dis Colon Rectum 2000; 43: 31-4.

6. Nienhuijs S, De Hingh I. Conventional versus LigaSure hemorrhoidectomy for patients with symptomatic hemorrhoids. Cochrane Database Syst Rev 2009; 2009; CD006761.

Received: 28.11 .2021

Accepted: 11.12 .2021 